\title{
CONSULTORÍA CONTABLE ADMINISTRATIVA: UNA NUEVA FORMA DE DIRIGIR UNA EMPRESA A TRAVÉS DE LA TECNOLOGÍA
}

\section{ARTÍCULO ORIGINAL}

SUMAR, Ramiro Rodrigues ${ }^{1}$

SUMAR, Ramiro Rodrigues. Consultoría contable administrativa: una nueva forma de dirigir una empresa a través de la tecnología. Revista Científica Multidisciplinar Núcleo do Conhecimento. Año 06, Ed. 07, Vol. 12, págs. 91 y 104. Julio de 2021. ISSN: 2448-0959, enlace de acceso: https://www.nucleodoconhecimento.com.br/administracion-de-empresas/la-

tecnologia, DOI: 10.32749/nucleodoconhecimento.com.br/administracion-deempresas/la-tecnologia

\section{RESUMEN}

Objetivo: Describir el impacto de los obstáculos y potencialidades que la tecnología ha traído a la consultoría contable. Pregunta: ¿Cómo puede la consultoría de contabilidad administrativa ayudar a una empresa a utilizar tecnologías? Métodos: El estudio es una revisión bibliográfica. Se realizó una búsqueda con los descriptores: tecnología; innovación; contabilidad; contabilidad en la plataforma de datos de Spell (Scientific Periodicals Electronic Library) en junio de 2021. La búsqueda dio como resultado 10 artículos. Resultados: los resultados trajeron como obstáculos: El creciente avance tecnológico, adaptarse al uso de estas nuevas tecnologías, Centralidad del papel del contador en los modelos tradicionales, Falta de conocimiento del software, Falta de mano de obra especializada, mal funcionamiento de la conexión, Mano de obra calificada. Y como potencialidades: Uso de diferentes

\footnotetext{
${ }^{1}$ Máster universitario en Contraloría, Especialista en Gestión Tecnológica, Administración Estratégica, Seguridad de la Información y Licenciado en Ciencias Contables.
}

$\mathrm{RC}: 93885$

Disponível em: https://www.nucleodoconhecimento.com.br/administracion-de- 
tecnologías digitales, Agilidad y ganancia de tiempo, Conveniencia, relación inseparable entre tecnología y contabilidad consultiva promoviendo un soporte ágil y de calidad a sus diversos usuarios, satisfacción del cliente, rapidez y flexibilidad y reducción de costes. Conclusiones: Se entiende que las Tecnologías de la Información influyen positivamente en el ejercicio de la contabilidad consultiva, aunque además de las ventajas tiene sus desventajas. Cabe mencionar que las tecnologías permiten el control de la información contable para la entrada y salida de materiales e insumos, así como su origen y destino final, evitando pérdidas innecesarias, incluyendo desviaciones, agilidad, flexibilidad y seguridad.

Palabras clave: Consultoría, Innovación, Medios tecnológicos, Seguridad de la información.

\section{INTRODUCCIÓN}

El vínculo entre contabilidad y tecnologías se ha perpetuado durante décadas, ya que, además de ser ciencia, la contabilidad es uno de los sistemas de información utilizados en la gestión empresarial más antigua, lo que hace que la contabilidad del pasado y la contabilidad del presente sean diferentes son las formas en que conducen a la gestión empresarial (FERREIRA et al., 2017).

La tecnología ha ido avanzando a lo largo de los años, innovando para que se satisfaga la necesidad del ser humano. Por lo tanto, al ser una herramienta ilimitada, la evolución de las tecnologías combinadas con la contabilidad aporta contribuciones relevantes al crecimiento de las organizaciones (SILVA et al., 2020).

El uso continuo de las redes informáticas y la combinación de diversos instrumentos contables favorecen el almacenamiento, registro, procesamiento y facilitan la realización del cruce de la información cada vez más rápido, lo que permite una toma de decisiones más segura dentro de la organización (MARION; RIBEIRO, 2017; DE ANDRADE SIMÕES et al., 2021).

$\mathrm{RC}: 93885$

Disponível em: https://www.nucleodoconhecimento.com.br/administracion-de- 
Actualmente, la búsqueda de información ha sido útil y al mismo tiempo relevante para el crecimiento de la organización. La transferencia de información al conocimiento, así como la aplicación oportuna del conocimiento en la práctica y la distribución de estos actos para el desarrollo de una empresa, son potencialidades de la tecnología en favor de la contabilidad consultiva para el emprendedor.

Sin embargo, muchos contadores no utilizan esta herramienta como una forma de ganar espacio, tiempo y retener a sus clientes, dejando claro que en algunas situaciones, las tecnologías para los contadores no se utilizan. En esta colocación, identificando la problematización del estudio en cuestión, está la pregunta que guió el tema de este estudio: ¿Cómo puede la consultoría contable administrativa ayudar a una empresa a utilizar las tecnologías?

El mercado laboral actual muchas veces motiva a las personas a reformularse en sus actividades profesionales, porque el sistema de educación universitaria básica y los nuevos conocimientos aún se vuelven pocos en vista de la práctica que el estudiante realizará después de graduarse.

Según Villa et al (2012, p.107), "los contadores tradicionales tienen la opinión de que los empresarios no buscan los conocimientos necesarios para gestionar su negocio, es decir, no toman cursos de mejora o formación en administración". Parafrasear esta cita del autor, indica que el empresario solo tiene el interés de satisfacer las exigencias de los impuestos, olvidando así el mercado competitivo, lo que hace que la empresa pierda espacio y no se desarrolle en su calidad.

En esta colocación, se observa la importancia del contador de asesoramiento. Este trabajo se suma a la justificación del investigador de entender por qué muchos contadores no utilizan la contabilidad consultiva para trabajar con sus clientes, ya que esta herramienta de ayuda sobre todo en tiempos de pandemia del nuevo coronavirus ejerce influencia para que la empresa no esté en riesgo de tener compromiso en sus ingresos en el competitivo mercado.

RC: 93885

Disponível em: https://www.nucleodoconhecimento.com.br/administracion-de- 
Por lo tanto, el tema cobra relevancia, porque con el advenimiento de las tecnologías, la contabilidad de las empresas tomó una dirección diferente a la contabilidad hace décadas y con esto, el contador ahora tiene la responsabilidad y el compromiso de hacer que su profesión sea identificada por la sociedad y los empresarios como una profesión que está avanzando para adaptarse a los medios tecnológicos con el fin de garantizar una mayor seguridad y confiabilidad a las organizaciones.

Por lo tanto, este trabajo tiene como objetivo general: Describir el impacto de los obstáculos y potencialidades que la tecnología ha traído a la consultoría contable.

\section{PROCESO METODOLÓGICO}

Este estudio es una revisión bibliográfica sistemática de carácter cualitativo. Según Sampaio y Mancini (2007) este tipo de estudio se lleva a cabo a través de la búsqueda de artículos relevantes para el tema investigado, basado en la síntesis de temas relevantes combinados con métodos sistematizados de investigación, análisis crítico, resumen de la información elegida y problemas importantes que requieren estudios adicionales.

Para llevar a cabo la investigación, se siguieron los siguientes pasos recomendados por Sampaio y Mancini (2007): Formulación de la pregunta guía del estudio; Localización de los estudios; Evaluación crítica de estudios; Recopilación de datos; Análisis de datos; Aspectos éticos.

De la construcción de una pregunta guía surgen algunos supuestos que orientan y/o delimitan este estudio, permitiendo así poner de relieve la aplicabilidad de las tecnologías en el universo de la consulta de los números y valores a los que se inserta el contador. La pregunta que guió el tema fue: ¿Cómo puede la consultoría de contabilidad administrativa ayudar a una empresa a utilizar las tecnologías?

RC: 93885

Disponível em: https://www.nucleodoconhecimento.com.br/administracion-de- 
El material fue seleccionado en las bases de datos: Spell (Scientific Periodicals Eletronic Library). La muestra de estudio consistió en artículos (investigación original; artículos de revisión; monografías, tesis y disertaciones). Se utilizaron como criterios de inclusión artículos en portugués e inglés, de 2016 a 2020, que están disponibles en su totalidad y de forma gratuita. Todos los artículos que se enmarcaron en estos criterios, que en su área de conocimiento cubrieron la contabilidad y tenían como título o temas abstractos relevantes para el uso de las tecnologías fueron enmarcados para la investigación. Se excluyeron otros artículos que no estaban incluidos o duplicados. Las palabras clave utilizadas para buscar estos artículos en Spell fueron: tecnología; innovación; contabilidad; contabilidad consultiva.

La evaluación crítica de las producciones seleccionadas e incluidas en el estudio dio lugar a interpretaciones y síntesis de los resultados, identificando la información científica presente en cada estudio y los factores relevantes para el estudio en cuestión sugiriendo pautas para futuras investigaciones. Para validar el estudio, el investigador deja en claro qué lagunas se encontraron en el estudio y qué caminos futuros pueden adoptar otros investigadores en su investigación científica. La información principal fue dispuesta en tablas, dejando en claro la información pertinente a los resultados de los artículos, componiendo los sujetos con la literatura pertinente al tema.

Los datos fueron recolectados durante junio de 2021. La recolección se realizó incluyendo los criterios de inclusión y exclusión de los artículos. Asimismo, destacando que la investigación también busca, a partir del análisis realizado, informar sobre la contabilidad consultiva y el uso de tecnologías. Se realizaron dos búsquedas intercalando las palabras clave.

Los datos encontrados en la literatura publicada ocurrieron de acuerdo con la propuesta de Ercole; Melo y Alcoforado (2014), en los que el revisor debe buscar

RC: 93885

Disponível em: https://www.nucleodoconhecimento.com.br/administracion-de- 
evaluar los resultados de manera imparcial, buscando explicaciones para los resultados diferentes o contradictorios en los diferentes estudios.

\section{ANÁLISIS DE RESULTADOS Y DEBATES}

Las búsquedas dieron como resultado un total de 343 resultados con las palabras clave. Se leyeron todos los títulos y resúmenes y se eliminaron los artículos que podían responder a los objetivos del estudio en cuestión, lo que resultó en 10 estudios.

Así, presentamos un cuadro con el título del artículo, autores, año de publicación (Referencia), objetivo del estudio y resultados encontrados.

Tabla 1 - Artículos seleccionados

\begin{tabular}{|c|c|c|}
\hline $\begin{array}{l}\text { ítulo } \\
\text { rtículo/Referencia }\end{array}$ & Metas & os \\
\hline $\begin{array}{l}\text { No caminho da } \\
\text { inovação: análise das } \\
\text { capacidades de } \\
\text { inovação de empresas } \\
\text { contábeis diante das } \\
\text { tecnologias digitais } \\
\text { Schiavi et al (2020). }\end{array}$ & $\begin{array}{l}\text { Examinar la capacidad } \\
\text { de innovación de las } \\
\text { oficinas de contabilidad } \\
\text { en el mercado brasileño, } \\
\text { en el uso de tecnologías } \\
\text { digitales, a partir de las } \\
\text { capacidades de } \\
\text { desarrollo tecnológico, } \\
\text { operación, gestión y } \\
\text { transacción. }\end{array}$ & $\begin{array}{l}\text { La contabilidad comienza en } \\
\text { el camino tradicional de la } \\
\text { innovación digital, } \\
\text { demostrando la calidad y el } \\
\text { valor que las soluciones } \\
\text { tecnológicas pueden generar } \\
\text { cuando se explotan en los } \\
\text { negocios y especialmente en } \\
\text { los procesos. Este aumento de } \\
\text { la tecnología provoca } \\
\text { cambios en los modelos de } \\
\text { negocio contables. }\end{array}$ \\
\hline $\begin{array}{l}\text { Indústria } 4.0 \text { e avanços } \\
\text { tecnológicos da área }\end{array}$ & $\begin{array}{lcc}\text { Analizar el } & \text { perfil } & \text { del } \\
\text { profesional } & \text { de } & \text { la }\end{array}$ & $\begin{array}{llr}\text { La mayoría de los } & \text { de } \\
\text { profesionales } & & \text { están }\end{array}$ \\
\hline
\end{tabular}

RC: 93885

Disponível em: https://www.nucleodoconhecimento.com.br/administracion-de- 
contábil:

percepções

expectativas

profissionais

Xavier; Carraro

Rodrigues (2020). perfil, contabilidad frente a los interesados en adaptarse a e avances tecnológicos, las nuevas tecnologías para dos con el objetivo de cumplir con los requisitos de explorar cómo se la función. Sin embargo, hubo comporta en relación con algunas resistencias a los las nuevas tendencias.

cambios, especialmente entre los profesionales menores de 35 años y en el área corporativa.

Características dos Identificar el valor El área contable enfocada en Diferentes Modelos de entregado al cliente de Sistemas de Información tiene Negócios Contábeis em los servicios contables, a sus entregas basadas en Relação às Áreas da través de la organización consultoría en procesamiento Contabilidade estructural y de datos, tecnología de la

Schiavi y Behr (2020). características de los información y sistemas modelos de negocio operativos. tradicionales, en relación con sus respectivas áreas de actividad en contabilidad.

As Contribuições da Compruebe

Contabilidade

Consultiva: Um Estudo de Caso/The

Contributions of Advisory

Accounting: A Case Study

Angelim y Barreto las Se comprobó que el percibir contribuciones la importancia de la promovidas por el contabilidad consultiva en el avance de la tecnología escenario actual, así como el en la contabilidad, así papel esencial de la como la contabilidad de tecnología en la profesión asesoramiento. contable. Los retos abordados por los encuestados incluyen la adaptación al uso de las

RC: 93885

Disponível em: https://www.nucleodoconhecimento.com.br/administracion-de- 
(2020).

nuevas

herramientas

tecnológicas, la necesidad de delinear estrategias para servir mejor al cliente y la necesidad de conocer la gestión y el negocio de cada cliente.

Impact of information Determinar el impacto de Los resultados de la technology on la tecnología de la investigación mostraron que accounting line of works información en la línea la tecnología de la (2020). sistema global. influencia sustancial en la línea de trabajo contable.

The impact of information Examine las ventajas de La contabilidad, como el and communication las aplicaciones de la lenguaje de los negocios y el technology (ICT) in the tecnología de la sistema de información, debe accounting system: información en la adaptarse a las nuevas advantages, contabilidad. tecnologías para servir a los applications, and usuarios de la información challenges

Al-Shafeay; Al_Dujaili y Al-Wattar (2020). financiera. En la era de la información, los requisitos del mercado para los graduados en contabilidad están cambiando progresivamente.

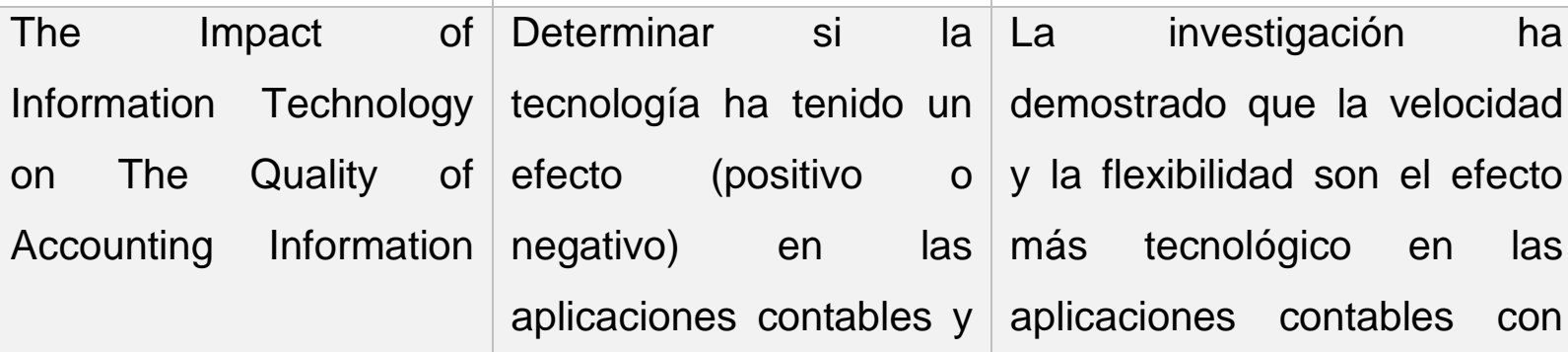

RC: 93885

Disponível em: https://www.nucleodoconhecimento.com.br/administracion-de- 
(SFAC NO 8, 2010)

Al Natour (2020). si la tecnología de la características mejoradas y información en la fiables.

contabilidad tiene un

efecto en la calidad de

los informes financieros

en Arabia Saudita.

A Consultoria Contábil Realizar un estudio La consultoría contable como Oportunidade de documental y presenta un factor de Negócios Profissionais - bibliométrico sobre rentabilidad financiera

Um Estudo Documental

Almeida et al (2018).

Consultoria

Organizacional como um Instrumento de Inovação em Arranjos Produtivos Locais

Silva; Ribeiro e Silva (2018).

Planejamento

Estratégico: Diagnóstico e Proposta de inte Intervenção por Meio de Consultoria

Gafuri; Rojo y Miura (2017). consultoría contable en cualitativa para las empresas, el periodo 2006-2016. aumentando así la ventaja competitiva para las mismas.

Analizar cómo las Las consultoras aportaron
prácticas de consultoría innovaciones para la mayoría organizacional pueden de las empresas, siendo el desencadenar la $89 \%$ en procesos, el $77 \%$ en innovación en los gestión empresarial, el 74\% Arreglos Productivos en productos y el $40 \%$ en Locales (APL). marketing.

Diagnosticar y presentar A través de un enfoque de propuestas de consultoría externa, una intervención a través de visión externa de la consultoría que organización, es contribuya al formato de indispensable adaptar las una planificación técnicas y herramientas al estratégica para una tipo de negocio que se empresa activa en el analiza, así como la actividad negocio del calzado y realizada, el tamaño de la

$\mathrm{RC}: 93885$

Disponível em: https://www.nucleodoconhecimento.com.br/administracion-de- 


$\begin{aligned} & \text { accesorios boutique de } \\ & \text { mujer. }\end{aligned}$
negociaciones.

Fuente: Autoría propia

Los artículos seleccionados identificaron obstáculos para el uso de tecnologías por parte del contador. La Tabla 2 proporciona una síntesis de estas dificultades a partir de la referencia del artículo seleccionado.

Cuadro 2 - Obstáculos encontrados

\author{
Título del artículo, Referencia \\ No caminho da inovação: análise das El creciente avance tecnológico \\ capacidades de inovação de empresas \\ contábeis diante das tecnologias \\ digitais \\ Indústria 4.0 e avanços tecnológicos Adaptarse al uso de estas nuevas \\ da área contábil: perfil, percepções e tecnologías \\ expectativas dos profissionais
}

Características dos Diferentes La centralidad del papel del contador en los Modelos de Negócios Contábeis em modelos de negocio contables Relação às Áreas da Contabilidade tradicionales, y los recursos tecnológicos siguen apoyando en este escenario.

As Contribuições da Contabilidade Adaptación al uso de las nuevas Consultiva: Um Estudo de Caso/The herramientas tecnológicas Contributions of Advisory Accounting:

A Case Study

Impact of information technology on Conserva los gustos detectables de tu accounting line of works pasado arraigados en el carácter y la integridad

RC: 93885

Disponível em: https://www.nucleodoconhecimento.com.br/administracion-de- 
The impact of information and Gerentes de proyectos inexpertos; Falta de communication technology (ICT) in the conocimiento del software; Contratos accounting system: advantages, incompletos; Falta de mano de obra applications, and challenges especializada.

The Impact of Information Technology Medios para hacer frente a problemas on The Quality of Accounting eléctricos, mal funcionamiento de la Information (SFAC NO 8, 2010) conexión a Internet y virus informáticos.

A Consultoria Contábil como Mayor duración y asignación de mayores Oportunidade de Negócios recursos y costos de ejecución.

Profissionais - Um Estudo Documental

Consultoria Organizacional como um Mano de obra calificada.

Instrumento de Inovação em Arranjos

Produtivos Locais

Planejamento Estratégico: Diagnóstico Falta de conocimiento del software.

e Proposta de Intervenção por Meio de

Consultoria

Fuente: Autoría propia

Se observa que hay varios obstáculos evidenciados en los artículos cuando se trata del uso de tecnologías para la consultoría contable. Estos obstáculos provienen principalmente de las dificultades de muchos profesionales que no se adaptan al uso de las tecnologías en su rutina, o porque el profesional no está debidamente calificado o calificado, o por miedo a los cambios tecnológicos.

Schiavi et al (2020) señala que las tecnologías deben ser vistas como un apoyo para apuntar a buenos resultados, especialmente en consultorías, sin embargo, el contador debe ser consciente de los avances tecnológicos que no son lentos, lo que permite que el profesional tenga miedo de utilizar la tecnología como un soporte específico para la consultoría.

$\mathrm{RC}: 93885$

Disponível em: https://www.nucleodoconhecimento.com.br/administracion-de- 
Xavier; Carraro y Rodrigues (2020) mencionan que la contabilidad consultiva debe dirigirse desde medios tecnológicos, ya que favorece una mayor seguridad, precisión y confiabilidad en los datos encontrados, sin embargo, para que esto suceda, le corresponde al contador estar al tanto de los cambios en los avances tecnológicos y adaptarse a todo el sistema, trayendo al cliente tecnologías avanzadas y de primera línea.

Schiavi y Behr (2020) y Angelim y Barreto (2020) informan que una parte de los profesionales de la contabilidad todavía tienen su dominio en la tradicionalidad de la ciencia contable, no permitiendo la presencia total de tecnologías de la mejor calidad en los servicios de consultoría contable, lo que refleja un retraso significativo en el descubrimiento de la toma de decisiones.

Hace necesario que el profesional sea consciente de las nuevas tendencias tecnológicas para la mejora de la empresa, un contador formado en el uso de tecnologías tiende a reducir el tiempo para lograr el equilibrio de la empresa, así como lograr pasar la fiabilidad en los datos recogidos.

Según Asuquo; Dan y Effiong (2020) la contabilidad consultiva trae la oportunidad de expandirse en todos los sectores de la contabilidad, ya sea de manera gerencial o financiera, lo que determina el trabajo de consultoría es la razón por la cual se trabajará en la consultoría, sin embargo, el arraigo de la contabilidad tradicional, crea un enorme obstáculo entre el contador y las tecnologías, donde este profesional todavía se siente más seguro en presencia del cliente en su oficina.

El desconocimiento de las tecnologías y sus beneficios siguen impregnando la vida de muchos profesionales. La falta de capacitación profesional, la falta de conocimiento sobre los programas, la mano de obra calificada entre otros obstáculos permiten que las tecnologías no ingresen a la consultoría contable y, por lo tanto, favorezcan de manera rápida, segura y efectiva, el análisis de datos $y$, en consecuencia, la toma de decisiones (AL-SHAFEAY; AL_DUJAILI; AL-WATTAR,

RC: 93885

Disponível em: https://www.nucleodoconhecimento.com.br/administracion-de- 
2020; ALMEIDA et al., 2018; SILVA; RIBEIRO; SILVA, 2018; GAFURI, ROJO; MIURA, 2017).

Sin embargo, los obstáculos no solo se relacionan con las dificultades de los contadores en el uso de las tecnologías, el mal funcionamiento de los programas, la lentitud de Internet, los virus en la computadora pueden influir en la no idoneidad del profesional para el medio de las tecnologías para ayudar en la contabilidad consultiva (AL NATOUR, 2020).

El uso de Software y Hardware requiere una intensa dedicación del profesional para poder saber utilizar las herramientas y con ello métodos actuales que pueden traer menores costos y mayores beneficios a la empresa. Teniendo en cuenta estos obstáculos en sus complejidades del entorno tecnológico, identifica las potencialidades que las tecnologías pueden aportar a la consultoría contable.

Tabla 3 - Potencial para la consultoría contable y el uso de tecnologías

\section{Título del artículo, Referencia}

No caminho da inovação: análise das capacidades de inovação de empresas contábeis diante das tecnologias digitais Indústria 4.0 e avanços tecnológicos Agilidad y ganancia de tiempo. da área contábil: perfil, percepções e Conveniencia. expectativas dos profissionais

$\begin{array}{lll}\text { Características dos Diferentes } & \text { El uso del uso de medios tecnológicos del } \\ \text { Modelos de Negócios Contábeis em } & \text { usuario como correos electrónicos, } \\ \text { Relação às Áreas da Contabilidade } & \begin{array}{l}\text { teléfono, entre otros para realizar } \\ \text { consultoría contable. }\end{array}\end{array}$

As Contribuições da Contabilidade Relación inseparable entre la tecnología y

RC: 93885

Disponível em: https://www.nucleodoconhecimento.com.br/administracion-de- 
Consultiva: Um Estudo de Caso/The la contabilidad consultiva promoviendo un Contributions of Advisory Accounting: soporte ágil y de calidad a sus diversos A Case Study usuarios.

Impact of information technology on Satisfaga a sus audiencias internas y accounting line of works externas dándoles servicios de calidad y rendimiento de forma continua y consistente.

The impact of information and Satisfacción del cliente; Agilidad; Fiabilidad. communication technology (ICT) in the accounting system: advantages, applications, and challenges

The Impact of Information Technology Velocidad y flexibilidad on The Quality of Accounting Information (SFAC NO 8, 2010)

A Consultoria Contábil como Propensos al riesgo a los resultados Oportunidade de Negócios esperados del trabajo a realizar

Profissionais - Um Estudo Documental

Consultoria Organizacional como um Nuevos métodos de organización del Instrumento de Inovação em Arranjos trabajo; Nuevas técnicas para la gestión del Produtivos Locais conocimiento; Cambios significativos en las relaciones con otras empresas

Planejamento Estratégico: Diagnóstico Reducción de costos, control de gestión e Proposta de Intervenção por Meio de con despliegue para los horarios de Consultoria compra.

Fuente: Autoría propia

Es evidente que la necesidad de profundizar en nuevos conceptos y conocimientos es de suma importancia para los profesionales de la contabilidad, para ello, se observa el potencial de las tecnologías en la consultoría contable. La contabilidad es

$\mathrm{RC}: 93885$

Disponível em: https://www.nucleodoconhecimento.com.br/administracion-de- 
considerada como una herramienta fundamental para apoyar el crecimiento de la empresa, sin embargo, la falta de conocimiento de las tecnologías por parte del contador hace que este conocimiento adquirido se identifique en la tradicionalidad de los roles.

Schiavi et al. (2020) mencionar varias potencialidades que las tecnologías pueden favorecer a la consultoría contable, la cita es: Uso de diferentes tecnologías digitales; actuar plenamente online para aportar más accesibilidad y agilidad en la prestación de servicios de contabilidad. Estas posiciones de los autores identifican la importancia de que la contabilidad y las tecnologías se muevan siempre unas junto a otras, lo que favorece la lealtad del contador a sus clientes.

Xavier; Carraro y Rodrigues (2020) corroboran con los autores anteriores sobre las potencialidades de las tecnologías para la consultoría contable y refuerzan que además de estas colocaciones también se pueden mencionar la agilidad, la ganancia de tiempo y la conveniencia tanto de la empresa como del contador.

Para Schiavi y Behr (2020) el uso de tecnologías para llevar a cabo la consultoría contable no solo está relacionado con temas de programas informáticos, teléfono, redes sociales, correos electrónicos, entre otras tecnologías puede ayudar al contador tradicional a realizar su consultoría sin necesariamente tener que trabajar con programas.

Según Angelim y Barreto (2020) las tecnologías ya tienen su participación en asesoría contable, sin embargo, la presencia de programas facilitadores para la realización de consultoría contable ha llevado a lo que se denomina tecnología de innovación, aportando agilidad y más confiabilidad en los datos presentados.

Según Asuquo; Dan y Effiong (2020) y Al-Shafeay; Al Dujaili y Al-Wattar (2020), la contabilidad consultiva tiene como objetivo proporcionar la información más fiable sobre la empresa y los resultados que obtiene, la satisfacción del cliente al brindar

RC: 93885

Disponível em: https://www.nucleodoconhecimento.com.br/administracion-de- 
servicios de calidad y rendimiento es lo que demuestra la diferencia de la presencia de la tecnología más avanzada para el contador.

Al Natour (2020) que las tecnologías son rápidas y flexibles, dos condiciones esenciales para el trabajo del contador que en su tiempo de consultoría se convierte en un enemigo para la toma de decisiones, ya que el mercado competitivo es cada vez más exigente en relación con las empresas.

Según Almeida et al. (2018) las tendencias para aumentar las ganancias de una empresa impactan directamente en el contador, lo que intensifica el conocimiento de la contabilidad sobre la asunción de riesgos. Las tecnologías actúan con el fin de garantizar un menor riesgo para el contador y una mejor rentabilidad para la empresa, lo que permite al cliente y al propietario de la empresa un servicio de calidad y en un tiempo ágil.

Silva; Ribeiro e Silva (2018) e Gafuri; Rojo y Miura (2017) destacan que además de proporcionar análisis precisos y seguros, las tecnologías también proporcionan métodos de organización del trabajo, gestión del conocimiento, mejor relación con otras empresas, control de costos, mejora en la toma de decisiones, entre otras potencialidades apuntando a una mayor productividad con menores costos en comparación con las técnicas tradicionales y manuales.

Se percibe que las tecnologías combinadas con la contabilidad consultiva tienen varias potencialidades que permiten que la contabilidad tenga su toma de decisiones para que la empresa no tenga problemas financieros o abandone el mercado competitivo, actuando sobre el problema evidenciado.

RC: 93885

Disponível em: https://www.nucleodoconhecimento.com.br/administracion-de- 


\section{CONSIDERACIONES FINALES}

Los obstáculos al uso de las tecnologías en la contabilidad consultiva siguen siendo motivo de preocupación, teniendo en cuenta que el profesional de la contabilidad debe tener como ayuda a la tecnología para mejorar su servicio y que la no aceptación del uso de las tecnologías puede provocar que este profesional sea descalificado y desacreditado de sus funciones.

El profesional contable debe tener en cuenta prácticas sostenibles para poder generar más beneficios financieros y operativos para las empresas, considerando que además del alto costo de adquisición y mantenimiento de todo el proceso de instalación tecnológica, existen gastos en cuanto a calificación y capacitación profesional.

La creciente demanda de programas informáticos influye directamente en la no aceptación de muchos profesionales, esto puede ser considerado de manera desventajosa para la contabilidad, ya que no existe un sistema único para realizar las actividades necesarias que la contabilidad necesita.

Pero el potencial que los avances tecnológicos aportan a la consultoría contable es inmenso, y estas potencialidades pueden generar impactos positivos en cuanto a la aceptación del uso de las tecnologías para lograr los objetivos en contabilidad. En este contexto, ha sido que las tecnologías facilitan la visión global del contador, en el que la percepción de un menor margen de error y el acceso a todos los datos de interés que pueden ser recopilados y/o facturados, encuentra en la tecnología un aliado para realizar un servicio de excelencia.

Se considera, por tanto, que el estudio logra su objetivo enumerado y se refiere como un vacío a la investigación realizada a partir de la consultoría contable en general, lo que abre vías para que se idealicen nuevas investigaciones extendiendo

RC: 93885

Disponível em: https://www.nucleodoconhecimento.com.br/administracion-de- 
este tema a una forma más restringida como la consultoría contable financiera o incluso gerencial.

\section{REFERENCES}

ALMEIDA, Leonardo Soares Francisco de et al. A Consultoria Contábil como Oportunidade de Negócios Profissionais-um Estudo Documental. Pensar Contábil, v. 20, n. $72,2018$.

AL NATOUR, Jihad Rebhee Abdel Qader et al. The Impact of Information Technology on The Quality of Accounting Information (SFAC NO 8, 2010). Turkish Journal of Computer and Mathematics Education (TURCOMAT), v. 12, n. 13, p. 885-903, 2020.

AL-SHAFEAY, Karrar Mohammed; AL_DUJAILI, Mohammed Jawad; AL-WATTAR, Yasir Mohammed Ali. The impact of Information and Communication Technology (ICT) In the accounting system: advantages, applications, and challenges. PalArch's Journal of Archaeology of Egypt/Egyptology, v. 17, n. 6, p. 15814-15825, 2020.

ANGELIM, Vitória Ribeiro; BARRETO, Tayssa Vieira. As Contribuições da Contabilidade Consultiva: Um Estudo de Caso/The Contributions of Advisory Accounting: A Case Study. ID on line Revista de Psicologia, v. 14, n. 52, p. 317331, 2020.

ASUQUO, Akabom I.; DAN, Nicholas O.; EFFIONG, Glory T. Impact of information technology on accounting line of works. International Journal of Recent Technology and Engineering, v. 9, n. 2, p. 1572-1577, 2020.

DE ANDRADE SIMÕES, Maervelym Pâmella et al. Benefícios do uso da tecnologia Blockchain como instrumento para a auditoria contábil. Revista ambiente contábilUniversidade Federal do Rio Grande do Norte-ISSN 2176-9036, v. 13, n. 1, p. 3953, 2021.

$\mathrm{RC}: 93885$

Disponível em: https://www.nucleodoconhecimento.com.br/administracion-de- 
ERCOLE, Flávia Falci; MELO, Laís Samara de; ALCOFORADO, Carla Lúcia Goulart Constant. Revisão integrativa versus revisão sistemática. Revista Mineira de Enfermagem, v. 18, n. 1, p. 9-12, 2014.

FERREIRA, Tiago Janes et al. Automação contábil: tecnologia em contabilidade sob a ótica da teoria institucional. XI Congresso ANPCONT. 3 a 6 de junho de 2017. Belo Horizonte, Minas Gerais.

GAFURI, Raquel Adriana Pin; ROJO, Cláudio Antonio; MIURA, Marcio Nakayama. Planejamento estratégico: diagnóstico e proposta de intervenção por meio de consultoria. Revista Capital Científico-Eletrônica (RCCe)-ISSN 2177-4153, v. 15, n. 1, p. 139-155, 2017.

MARION, JOSE CARLOS; RIBEIRO, OSNI MOURA. Introdução à contabilidade gerencial. Saraiva Educação SA, 2017. Disponível em: https://books.google.com.br/books?hl=pt-

$B R \& \mid r=\& i d=k D 1 n D w A A Q B A J \& o i=f n d \& p g=P T 5 \& d q=$ Introdu\%C3\%A7\%C3\%A3o+\%C 3\%A0+contabilidade+gerencial.+\&ots=JjrSnq2mD0\&sig=vcUiQXgi1UkNhLanfgWU67 QVgas\#v=onepage\&q=Introdu\%C3\%A7\%C3\%A30\%20\%C3\%A0\%20contabilidade\% 20gerencial.\&f=false

SAMPAIO, Rosana Ferreira; MANCINI, Marisa Cotta. Estudos de revisão sistemática: um guia para síntese criteriosa da evidência científica. Brazilian Journal of Physical Therapy, v. 11, p. 83-89, 2007.

SCHIAVI, Giovana Sordi; BEHR, Ariel. Características dos diferentes modelos de negócios contábeis em relação às áreas da Contabilidade. REUNIR Revista de Administração Contabilidade e Sustentabilidade, v. 10, n. 3, p. 47-59, 2020.

$\mathrm{RC}: 93885$

Disponível em: https://www.nucleodoconhecimento.com.br/administracion-de- 
SCHIAVI, Giovana Sordi et al. No caminho da inovação: análise das capacidades de inovação de empresas contábeis diante das tecnologias digitais. Revista Brasileira de Gestão de Negócios, v. 22, p. 381-405, 2020.

SILVA, Gustavo Oliveira et al. O impacto da tecnologia na profissão contábil sob perspectivas de pessoas com formação e/ou experiência profissional na área. Contribuciones a las Ciencias Sociales, n. 72, p. 3, 2020.

SILVA, Fabiana Ferreira; RIBEIRO, Ana Regina Bezerra; SILVA, Washington Ferreira. Consultoría Organizacional como um Instrumento de Inovação em arranjos produtivos locais. Revista Organizações em Contexto, v. 14, n. 27, p. 29-52, 2018.

VILLA, Patricia et al. O monólogo contábil: uma análise do uso da contabilidade gerencial em micro e pequenas empresas à luz do processo comunicacional segundo Bakhtin". Monografia para obtenção de título de mestrado em contabilidade. UFPR, 2012.

XAVIER, Leonardo Montes; CARRARO, Wendy Beatriz Witt Haddad; RODRIGUES, Ana Tércia Lopes. Indústria 4.0 e avanços tecnológicos da área contábil: perfil, percepções e expectativas dos profissionais. Contexto. Porto Alegre, RS. Vol. 20, n. 45 (maio/ago. 2020), p. 34-50, 2020.

Enviado: Julio de 2021.

Aprobado: Julio de 2021.

RC: 93885

Disponível em: https://www.nucleodoconhecimento.com.br/administracion-de- 